\title{
Research Paper \\ Perceived Family Functioning and Suicidal Ideation Among University Students: Hopelessness as a Moderator
}

\section{Asadollah Akbari ${ }^{1}$, *Leili Panaghi ${ }^{2}$, Mojtaba Habibi ${ }^{3}$, Mansoure Alsadat Sadeghi ${ }^{4}$}

1. MSc, Department of Clinical Psychology, Faculty of Education and Psychology, University of Shahid Beheshti, Tehran, Iran.

2. MD of Community Medicine, Associate Professor, Family Research Institute, University of Shahid Beheshti, Tehran, Iran.

3. PhD of Health Psychology, Assistant Professor, School of Behavioral Sciences and Mental Health (Tehran Institute of Psychiatry), Iran University of Medical Sciences,

Tehran, Iran

4. PhD of Public Psychology, Assistant Professor, Family Research Institute, University of Shahid Beheshti, Tehran, Iran.

Received: 07 Jun. 2016

Accepted: 03 Oct. 2016

Key words:

Suicidal ideation,

Family function

Hopelessness
Citation: Akbari A, Panaghi L, Habibi M, Sadeghi MA. [Perceived Family Functioning and Suicidal Ideation Among University Students: Hopelessness as a Moderator (Persian)]. Iranian Journal of Psychiatry \& Clinical Psychology. 2017; 22(4):284-291. https://doi.org/10.18869/nirp.ijpcp.22.4.284

doi : $:$ https://doi.org/10.18869/nirp.ijpcp.22.4.284

\begin{abstract}
A B S TRACT
Objectives The present study aims to determine whether the association between family function and suicidal ideation on one hand and correlation of family functions and hopelessness on the other hand can be supposed that hopelessness acts as a moderator in the association between family functions and suicidal ideation.

Methods For this, 373 accessible Shahid Beheshti students were selected and measured with self-reporting means. After performing Family Assessment Devices, Beck Hopelessness Scale and Beck scale for suicidal ideation, the gathered data were analyzed through simple correlation and hierarchical regression by SPSS 19.

Results There are significant positive correlations between understanding total family function and hopelessness, family function and suicidal ideation and also between hopelessness and suicidal ideation of students. Therefore, hopelessness has a moderating role in the association between understanding family function and suicidal ideation.

Conclusions The association between family function and suicidal ideation increases at higher levels of hopelessness while in lower levels of hopelessness, this association is not significant.
\end{abstract}

* Corresponding Author:

Address: Family Research Institute, University of Shahid Beheshti, Tehran, Iran.

Tel: +98 (21) 29902389

E-mail: panaghi@sbu.ac.ir 


\title{
كاركرد خانواده ادراكشده و افكار خودكثى دانشجويان: نقش تعديل كننده ناميدى
}

\author{
اسدالله اكبرى'، "ليلى يناغى"، مجتبى حبيبى"، منصورهسادات صادقى

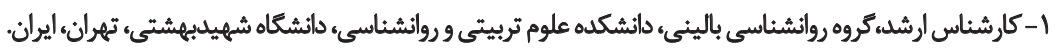

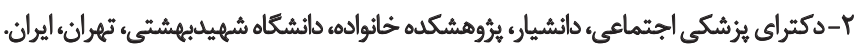

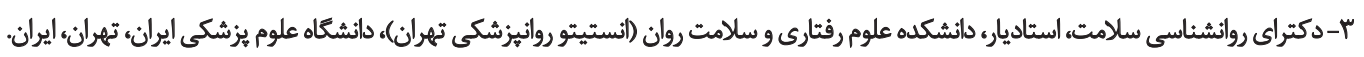

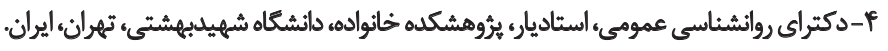

\begin{abstract}
حكيد

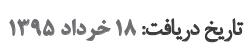

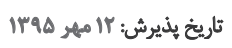

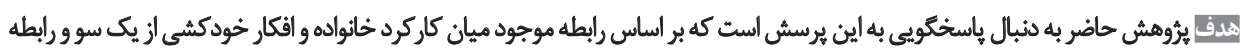

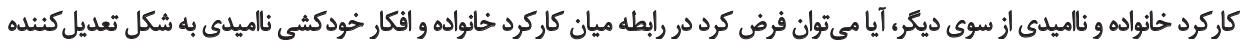

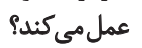

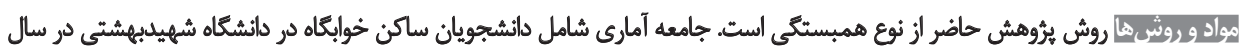

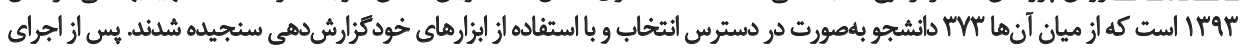

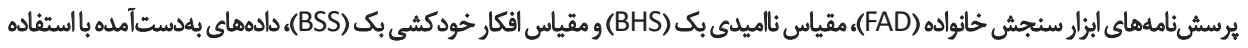

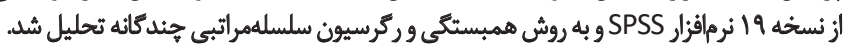

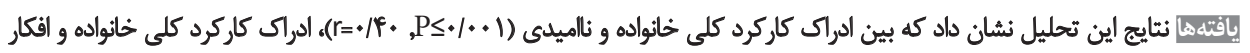

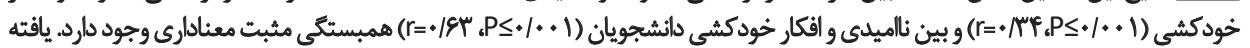

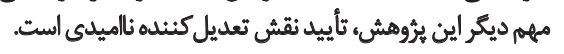

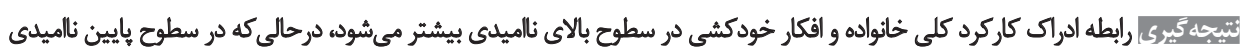

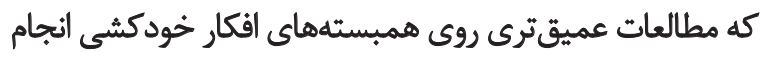

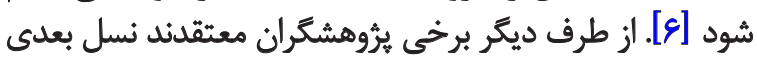

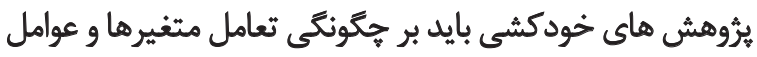

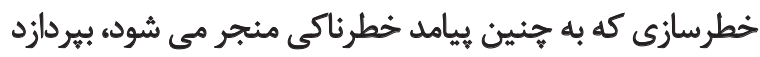

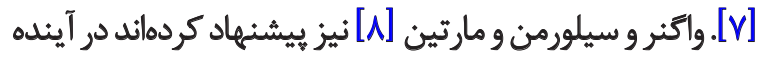

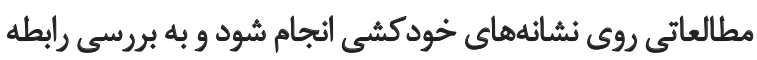

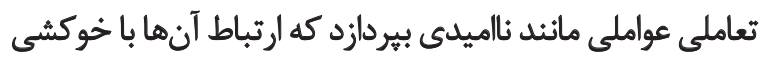

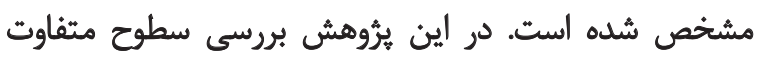
نالميدى به عنوان متغير تعديل شنثنده و ارتباط آن بـ بأ كاركرد

2. Suicide attempt

3. completed suicide

دانشجويان در زمره گروههايى هستند كه به دليل ماهيت

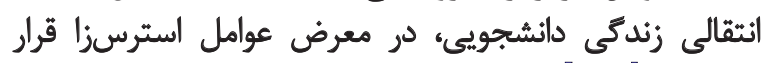

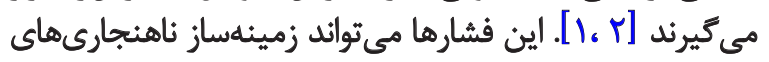

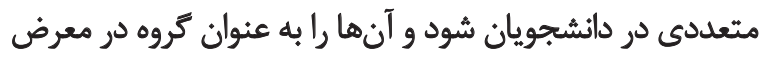

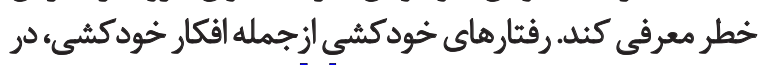

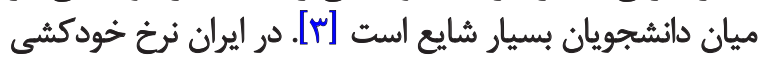

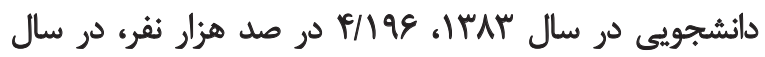

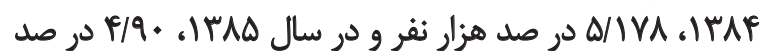

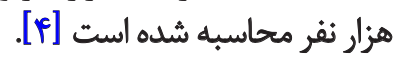

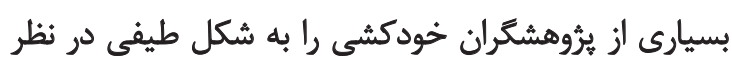

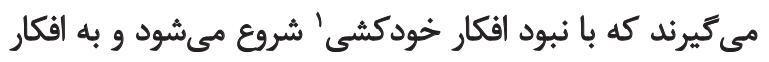

1. Suicidal ideation

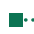

: نويسئده مسئول:

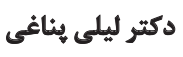

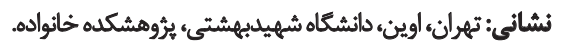

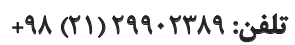
panaghi@sbu.ac.ir يست الكترونيكي 


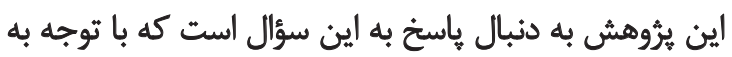

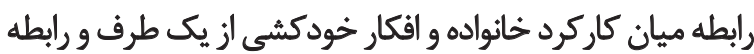

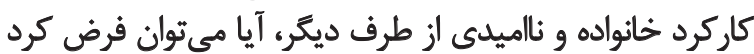

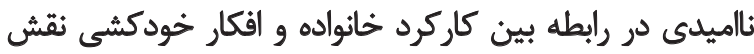

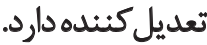
ووشي

يُروهش حاضر از نوع توصيفى همبستّى است. جامعه آمارى

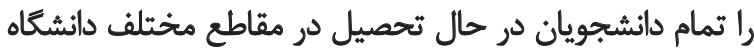

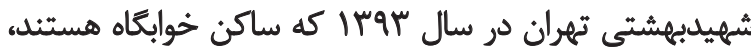

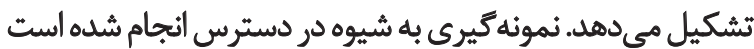

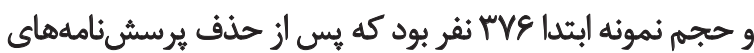

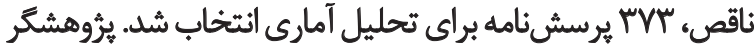

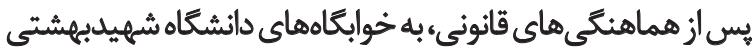

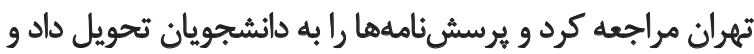

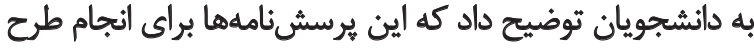

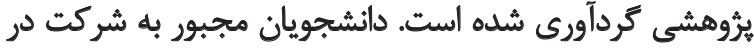

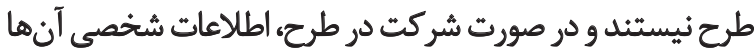

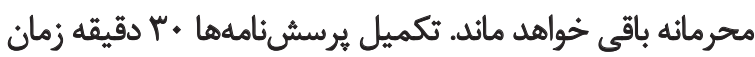

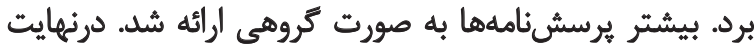

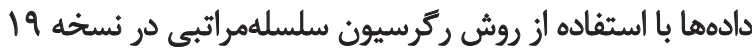

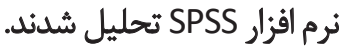

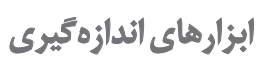

ابزار سنجش خانواده"

اين يرسشنامه شامل •ع سؤال است كه ايشتاين و بالدوين

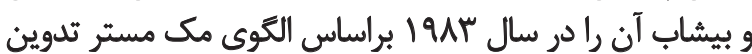

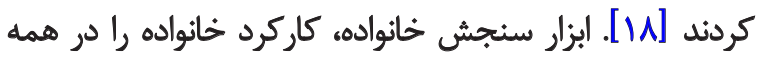

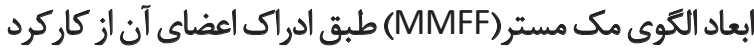

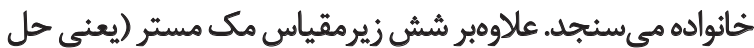

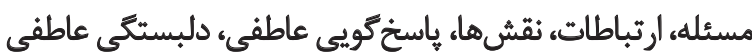

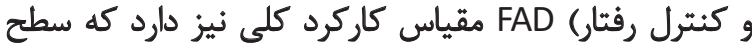

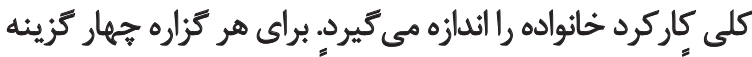

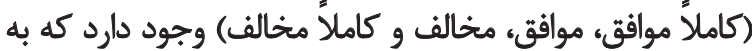

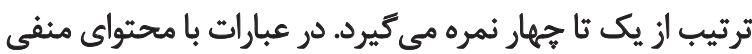
اين نمرهكذارى برعكسمى شود.

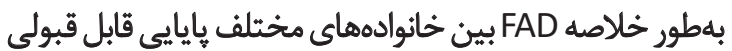

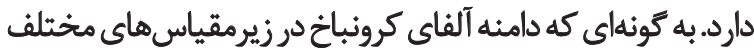

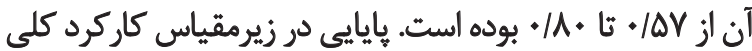

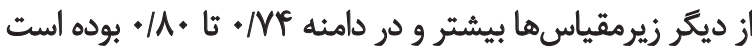

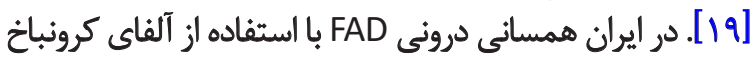

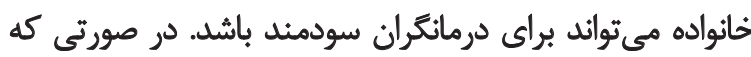

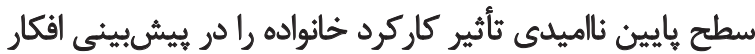

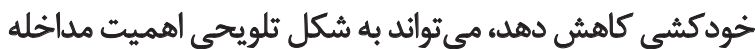
در اين متغير را به درمانكران نشان دهان بهد.

يكى از اساسىترين عوامل شناختى خطرساز مرتبط بارئ افكار

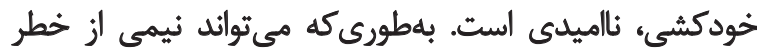

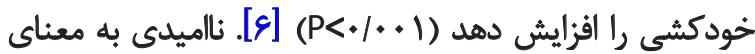

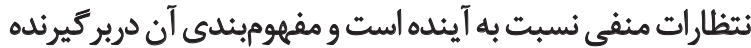

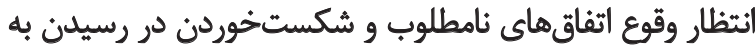

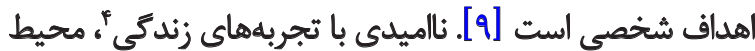

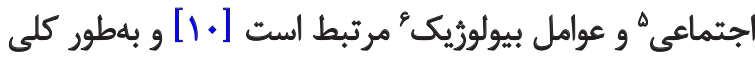

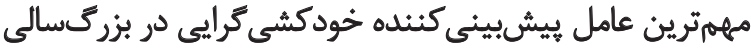

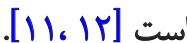

كاركرد خانواده به كيفيت زندكى خانوادكى در سطح سيستمى

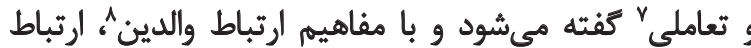

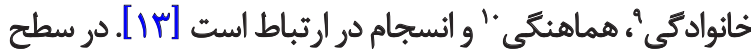

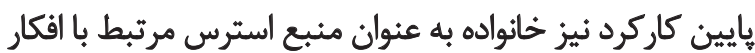

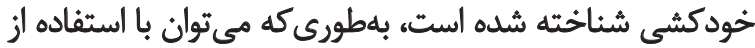

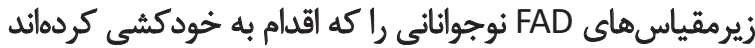

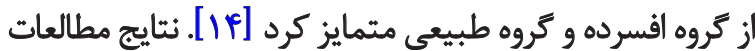

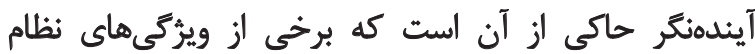

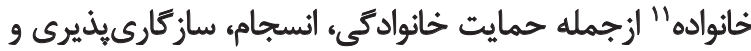

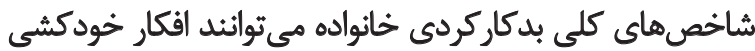

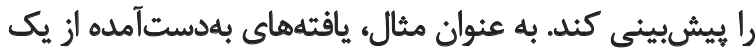

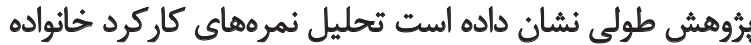

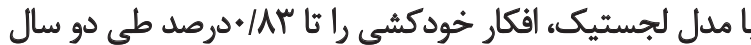

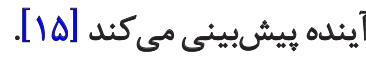

كاركرد خانواده ادراكشده به عنوان عامل خطرساز خانوادتى

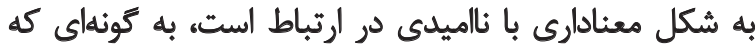

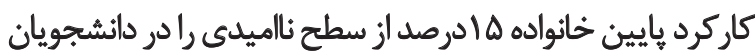

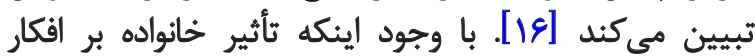

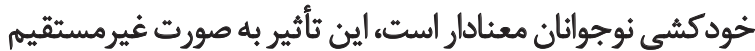

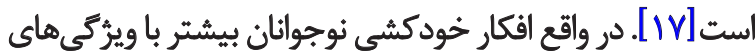

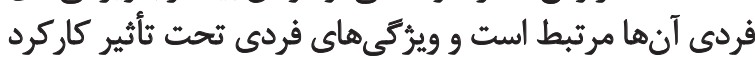

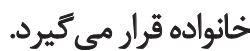

4. Life experience

5. Social-environmental

6. Biological factors

7. Dyadic

8. Parental concern

9. Family communication

10. Harmony

11. Whole family system 


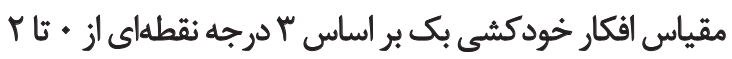

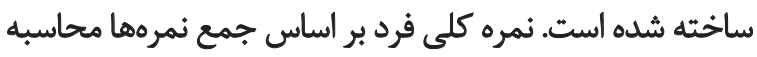

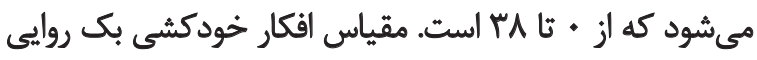

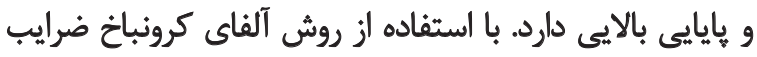

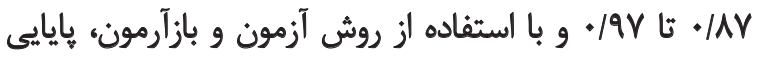

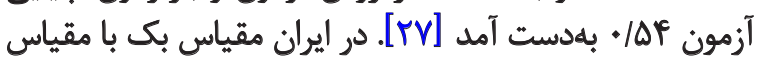

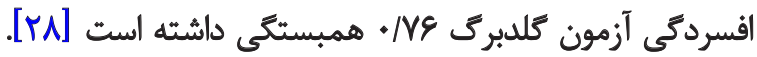

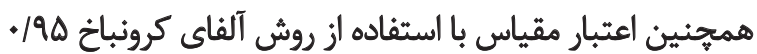

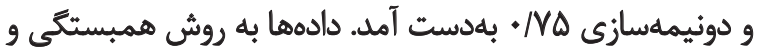

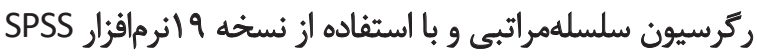

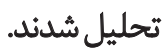

يافتهها

در جدول شماره ا آمار توصيفى و ضرايب همبستكى بين متغيرهاى بثروهش كزارش شده است.

براي تعيين اثر تعديل كنئده ناميدى، از نمرهماي استاندارد

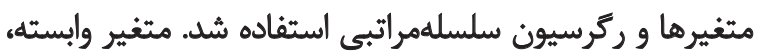

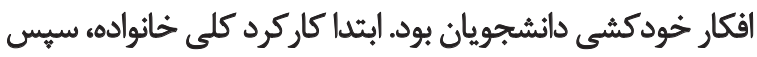

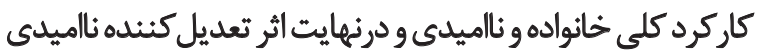

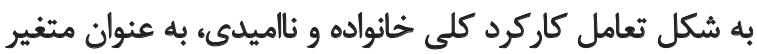

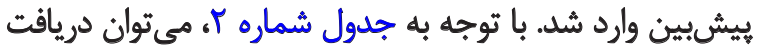

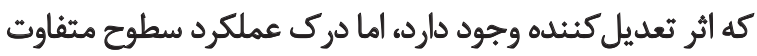

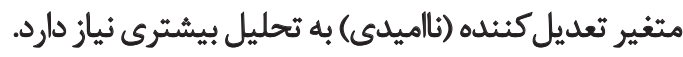

در مرحله بعد، يُشبينى افكار خودكشى دانشجويان از طريق

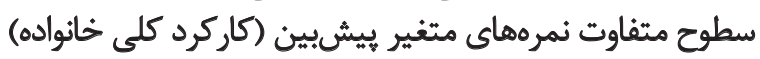

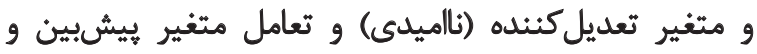
تعديل كنئده (كاركرد كلى خانواده ×ناميدى) بالي استفاده از نمودار

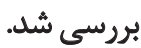

\section{$Y=a+(b 1+b 3 M) X+b 2 M$}

بادر نظر كرفتن سطوح مختلف اين متغيرها نه نقطه به دست

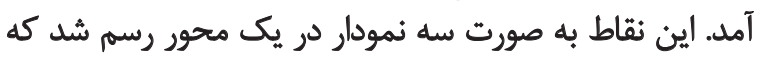
افكار خودكشى دانشجويان در سه سطح (بالاو مثتوسط و وايينين
و اعتبار بازآزمايى آن با فاصله يك هفته تا ده روز محاسبه شد كه

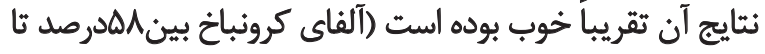

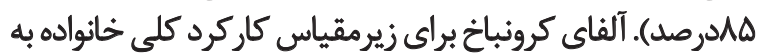

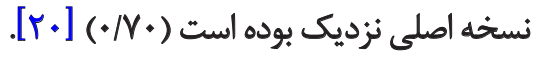

\section{مقياس نااميدى بك" (BHS)}

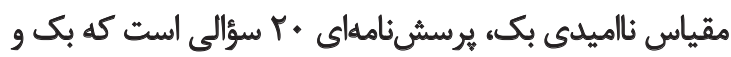

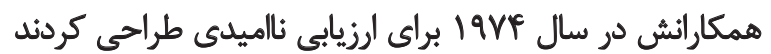

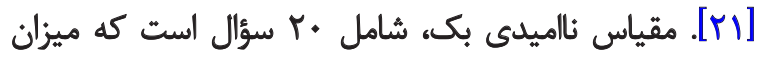

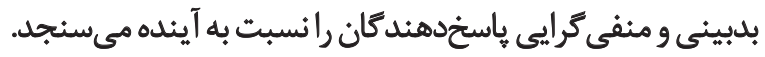

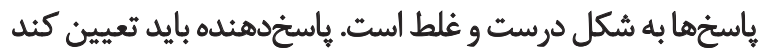

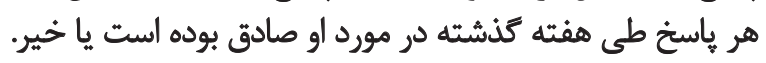

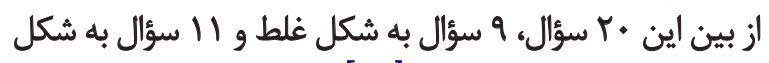

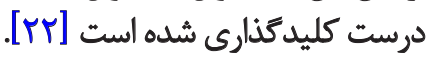

ثرئرهشهاى بسيارى روايى بالا و واياييى قابل قبول مقياس

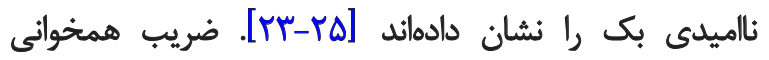

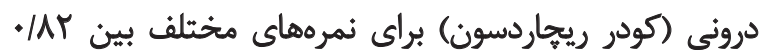

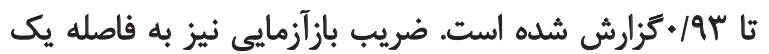

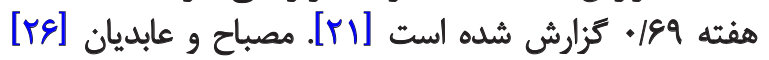

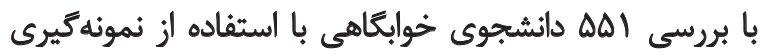

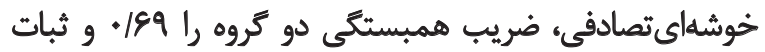

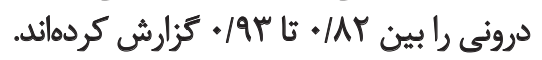

\section{مقياس افكار خودكشى بك" (BSS)}

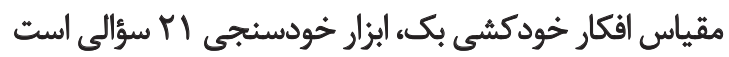

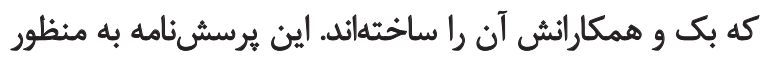

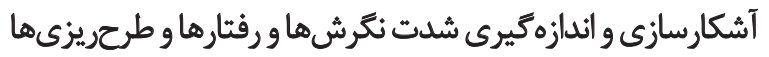

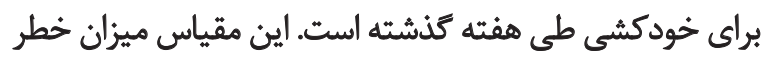

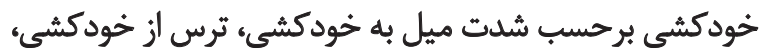

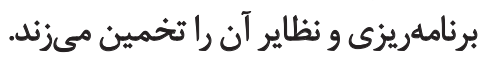

13. Beck Hopelessness Scale

14. Beck Scale for Suicide Ideation

جدول ا. يافتهماي توصيفى.

\begin{tabular}{|c|c|c|c|c|c|}
\hline كاركرد خانواده & نالميدى & أفكار خودكشي & ييشينه-كمينه & ميانعين (انحراف معيار) & مثيغير \\
\hline.$/ m p e n$ & . Iexen & 1 & שו & $r / P(\varphi / * r)$ & افكار خودكشى \\
\hline$. / 4+\cdots$ & 1 & . Igeos" &.-19 & $F / \cdot \Lambda(r / \wedge \Delta)$ & نالميدى \\
\hline 1 & 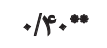 &.$/ m e * *$ & $1-f$ & $T / \cdot H(\cdot / \Delta T)$ & كاركرد خانواده \\
\hline.$/ M F$ &.$/ 1 f$ &.$/ 1 r^{*}$ & M-re & $M / \Delta(T / A))$ & سن \\
\hline
\end{tabular}


جدول ז. اثر تعديل كنيده ناميدى بر كاركرد كلى خائواده و افكار خودكشى دانشجويان با استفاده از ركرسيون سلسلهمراتبى.

\begin{tabular}{|c|c|c|c|c|c|}
\hline سطح مغنادارى & $\mathbf{t}$ & ضرايب اسثائدارد شده (Beta) & وزن ركريون استاندارد نشده (B) & 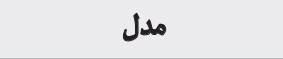 & 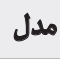 \\
\hline$* / \mathrm{V}$ & $-1 / / \mu$ & & -.1 .8 & (ثابت) & \multirow{2}{*}{1} \\
\hline$+1+\infty$ & $8 / 91$ &.$/ \pi$ &.$/ 4 q$ & كاركرد كلى خانواده & \\
\hline.$/ P$ & $-1 / 4 a$ & & -.100 & (ثابت) & \multirow{3}{*}{ r } \\
\hline $.1+1$ & T/FV &.$/ 11$ & .1 .9 & كاركرد كلي خانواده & \\
\hline$*+.1$ & $|r / T|$ & .109 & $\cdot(\Delta)$ & نالميدى & \\
\hline$\%$ & $-r / T V$ & & $-+/ N r$ & (ثابت) & \multirow{4}{*}{$r$} \\
\hline$\% \Delta$ & T/As &.$/ 1 r$ & .111 & كاركرد كلى خانواده & \\
\hline$\%$ & 1.188 & .10 &.$/ p p$ & نالميدى & \\
\hline.+1 & F/VD & $\cdot / M I$ &.$/ M$ & كاركرد كلى خاثواده× ناميلى & \\
\hline
\end{tabular}

به ميزان بr/• افكار خودكشى در او افزايش مي يابد.

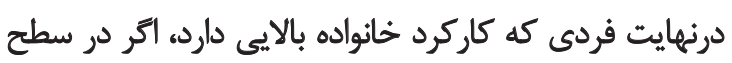

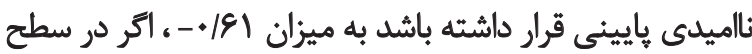

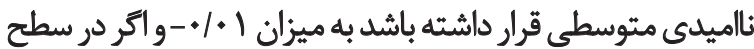

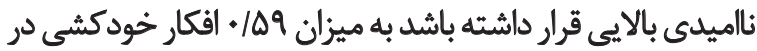

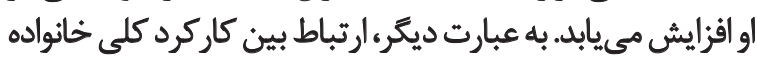

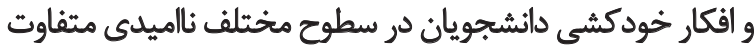

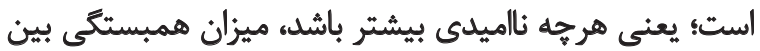
كاركرد كلى خانواده و افكار خودكشى بيشت بيشتر مى بشود.

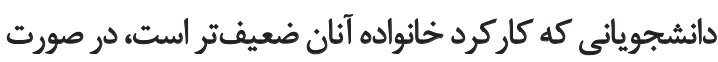

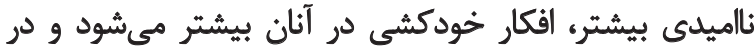

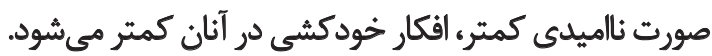

متغير ملاك: افكار خودكشى (استانداردشده).

از متغير بيشبين (كاركرد كلى خانواده) و متغير تعديل كننده

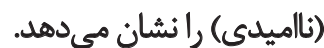

همانطور كه در تصوير شماره ا 1 ديده مىشود، هر يك إنى

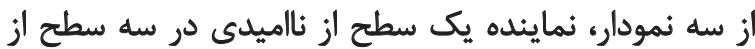

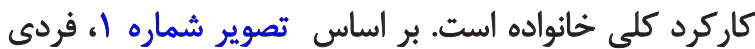

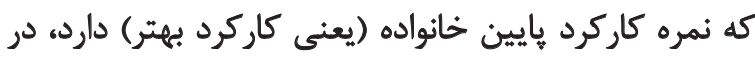

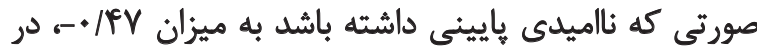

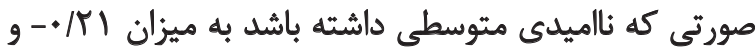

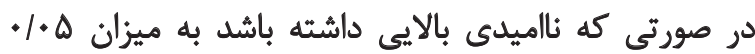

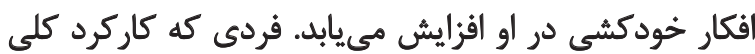

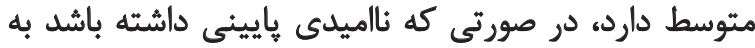

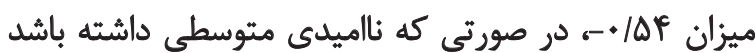

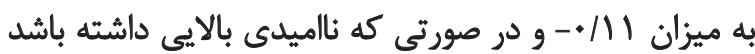

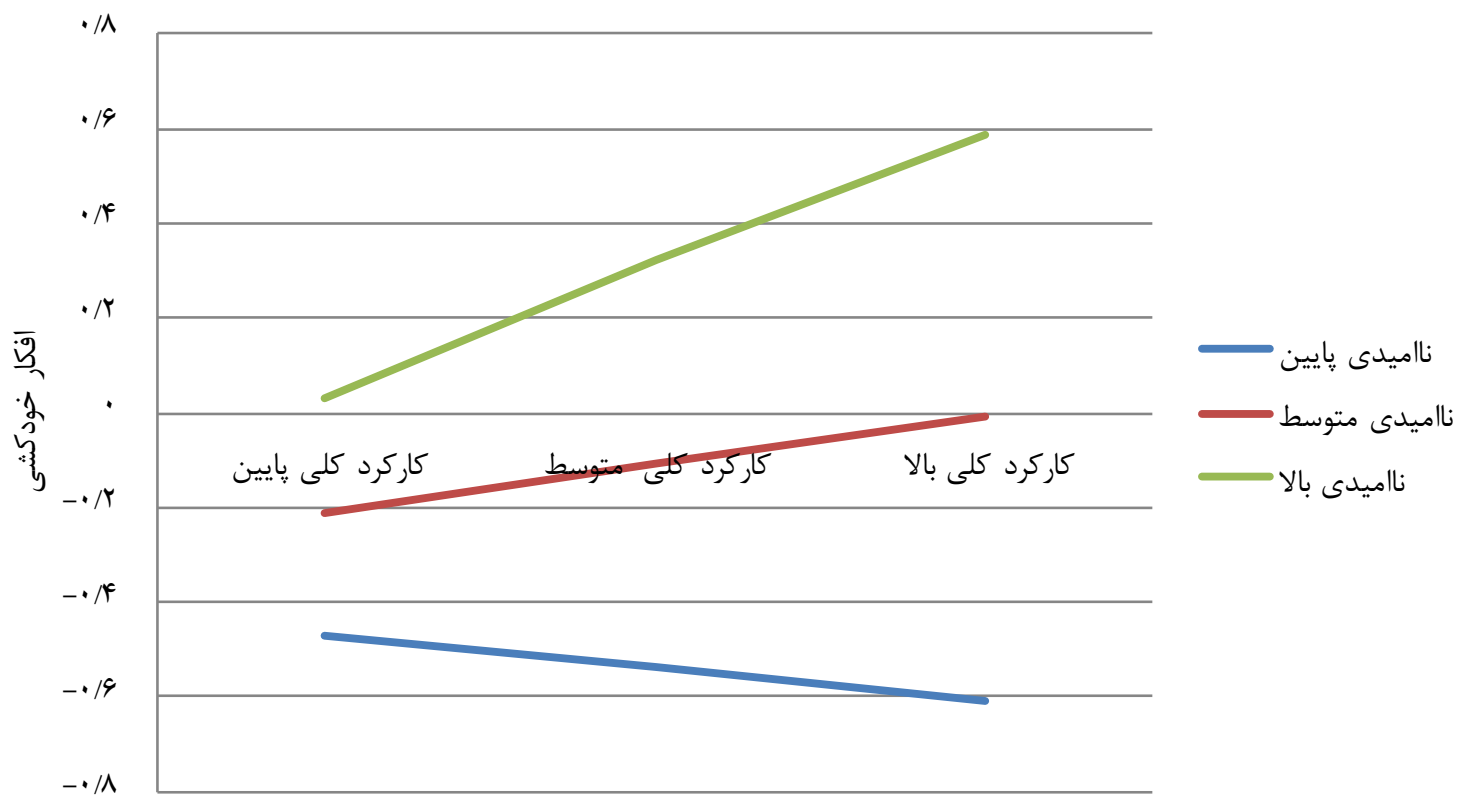


تفكر منفى مىشود كه افكار خودكشى رادر بيى دارد.

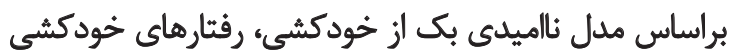

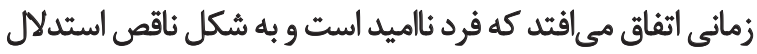

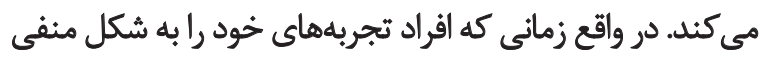

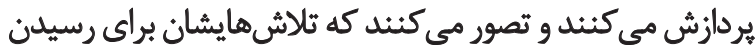

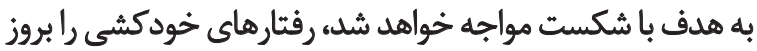

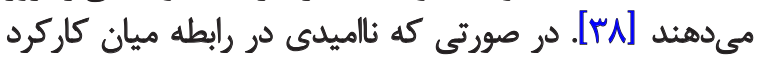

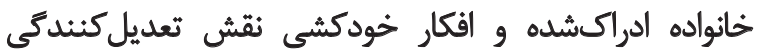

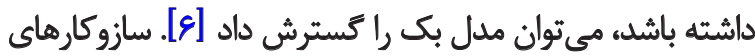

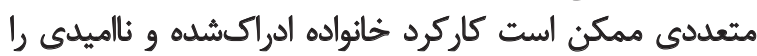

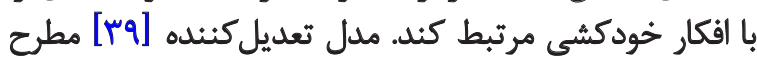

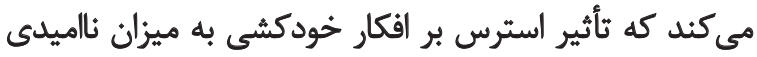

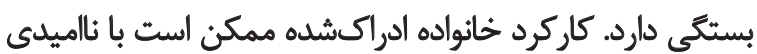

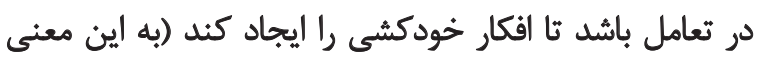

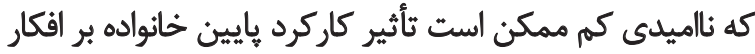
خود كشى راكاهش دهد).

ميثتوان كفت ارتباط ميان كاركرد كلى خانواديه با افكار

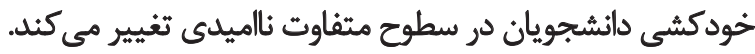

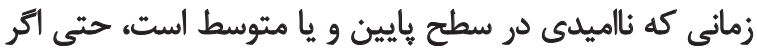

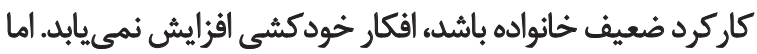

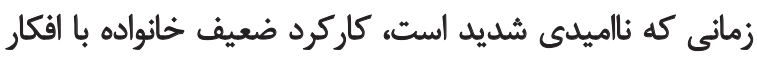

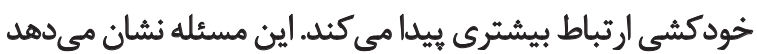

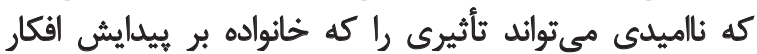

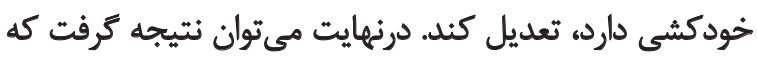

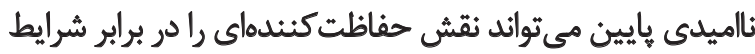
بيرونى مانند داشتن خانواده آشفته داشته باشند.

بثروهش حاضر محدوديت هايى داشت. نمونه ميرى در بروهش

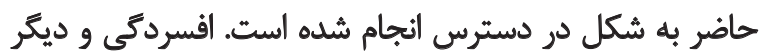

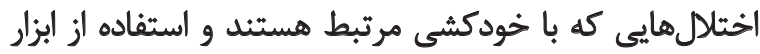

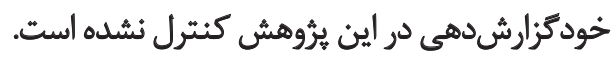

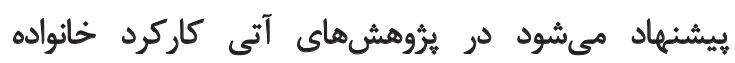

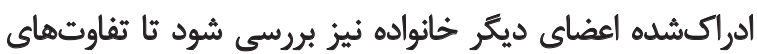

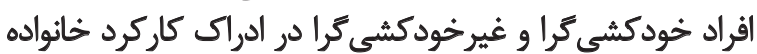

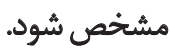

تيجيجه كَيرى

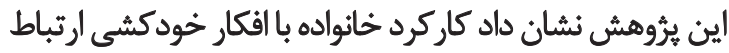

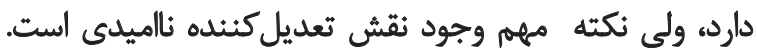

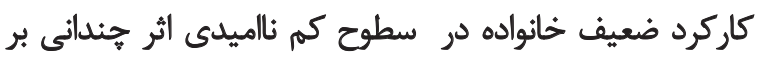

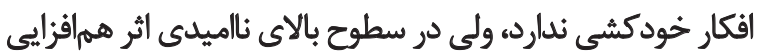

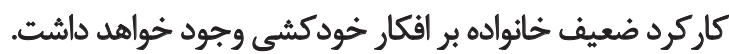

ب

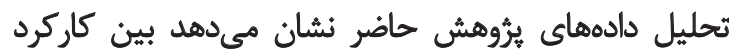

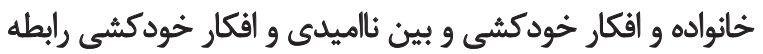

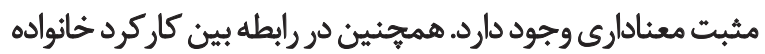

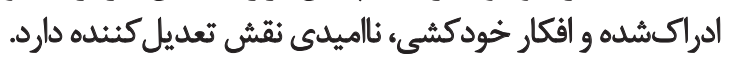
وجود رابطه مثبت معنادار بين كاركرد كلي خانواده ادراكشده

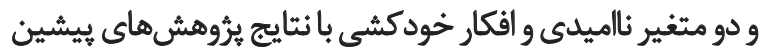

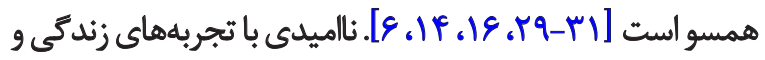

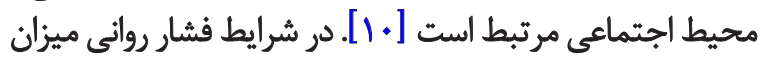

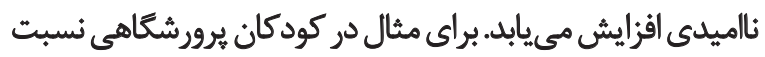

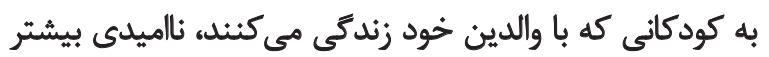

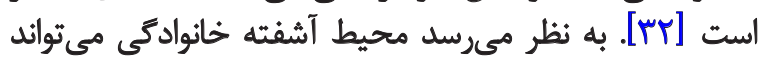

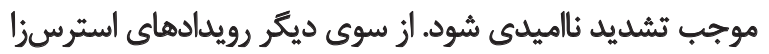

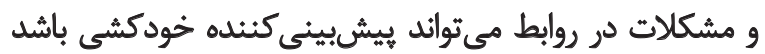

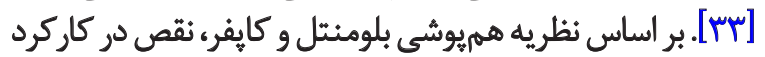

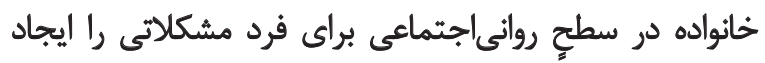

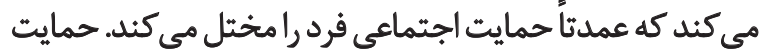

اجتماعى ضعيف، افكار خودكشى را تقويت مي كند [هايت]

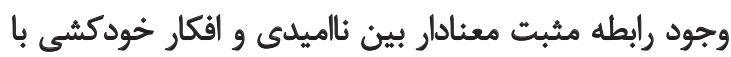

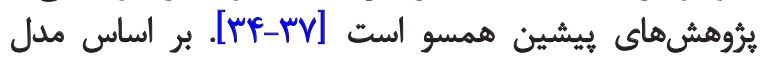

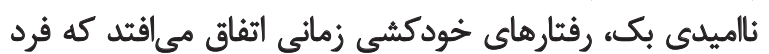

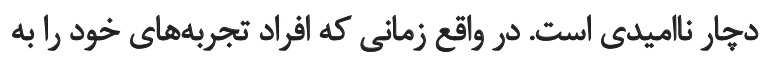

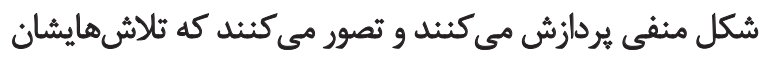

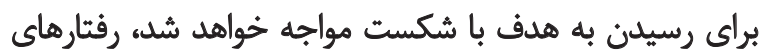

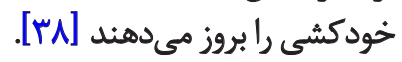

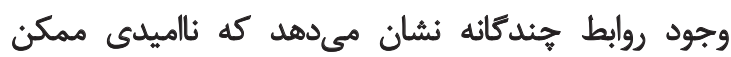

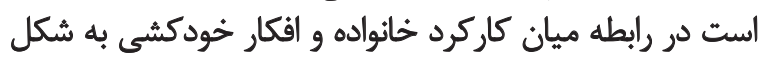

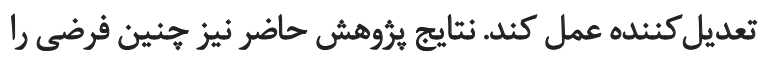

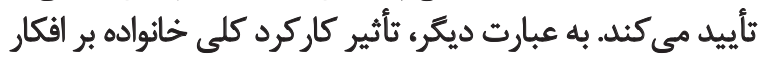

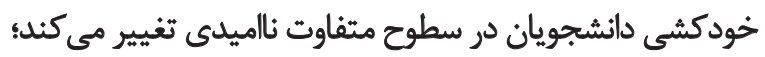

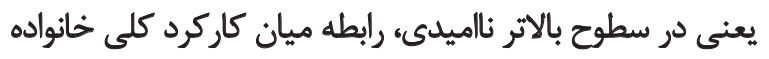

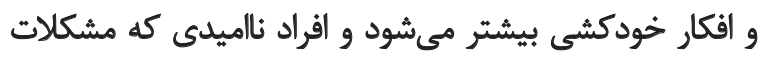

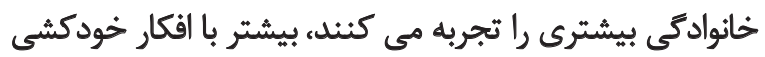

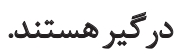

در ثبيين اين يافته مىتوان كفت با وجود اينكان ائكه تأثير خانواده

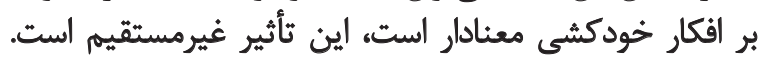

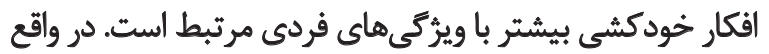

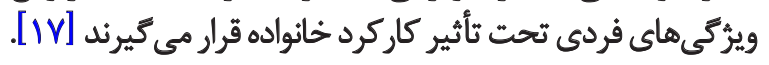

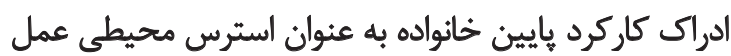

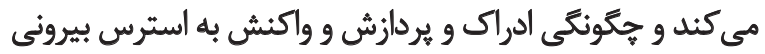

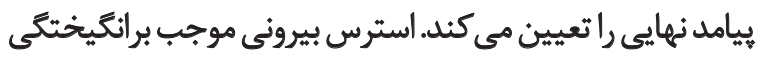




\section{References}

[1] Greenberger E, McLaughlin CS. Attachment, coping and explanatory Style in late adolescence. Journal of Youth and Adolescence. 1998; 27(2):121-139. doi: 10.1023/a:1021607627971

[2] McCarthy CJ, Moller NP, Fouladi RT. Continued attachment to parents: Its relationship to affect regulation and perceived stress among college students. Measurement \& Evaluation in Counselling and Development. 2001;33(4):198-212.

[3] Chang EC. Cultural differences, perfectionism, and suicidal risk in a college population: Does social problem solving still matter? Cognitive Therapy \& Research. 1998; 22(3):237-254. doi: 10.1007/ s10608-015-9711-7

[4] Panaghi L, Ahmadabadi Z, Peiravi H, Abolmasoomi FZ. [Suicide trend in university students during 2003 to 2008 (Persian)]. Iranian Journal of Practice in Clinical Psychology. 2010; 16(2):8798

[5] Westefeld JS, Range LM, Rogers JR, Maples MR, Bromley JL, Alcorn J. Suicide: An Overview. Counselling Psychologist. 2000; 28(4):445-510. doi: 10.1177/0011000000284002

[6] Kwok SY. Perceived family functioning and suicidal ideation: Hopelessness as mediator or moderator. Nursing Research. 2011; 60(6):422-429. doi: 10.1097/nnr.0b013e31823585d6

[7] Dour HJ, Cha CB, Nock MK. Evidence for an emotion-cognition interaction in the statistical prediction of suicide attempts. Behaviour Research \& Therapy. 2011; 49(4):294-8. doi: 10.1016/j. brat.2011.01.010

[8] Wagner BM, Silverman MA, Martin CE. Family factors in youth suicidal behaviors. American Behavioral Scientist. 2003; 46(9):1171-91. doi: 10.1177/0002764202250661

[9] Beck AT, Steer RA, Kovacs M, Garrison B. Hopelessness and eventual suicide: A 10-year prospective study of patients hospitalized with suicidal ideation. American Journal of Psychiatry. 2011; 142(5):559-63. doi: 10.1176/ajp.142.5.559

[10] Durant T, Mercy J, Kresnow MJ, Simon T, Potter L, Hammond WR. Racial differences in hopelessness as a risk factor for a nearly lethal suicide attempt. Journal of Black Psychology. 2006; 32(3):285-302. doi: 10.1177/0095798406290468

[11] Rotheram-Borus MJ, Trautman PD. Hopelessness, depression, and suicidal intent among adolescent suicide attempters. Journal of the American Academy of Child \& Adolescent Psychiatry. 1988; 27(6):700-704. doi: 10.1097/00004583-198811000-00006

[12] Van Heeringen C, Audenaert K, Van Laere K, Dumont F, Slegers G, Mertens J, Dierckx RA. Prefrontal 5-HT< sub $>2 \mathrm{a}<$ / sub> receptor binding index, hopelessness and personality characteristics in attempted suicide. Journal of Affective Disorders. 2003; 74(2):149-158. doi: 10.1016/s0165-0327(01)00482-7

[13] Shek, DTL. Assessment of family functioning in Chinese adolescents: The Chinese version of the Family Assessment Device. Research on Social Work Practice. 2002; 12(4):502-524. doi: $10.1177 / 1049731502012004003$

[14] King CA, Segal HG, Naylor M, Evans T. Family functioning and suicidal behavior in adolescent inpatients with mood disorders. Journal of the American Academy of Child \& Adolescent Psychiatry. 1993; 32(6):1198-1206. doi: 10.1097/00004583199311000-00013

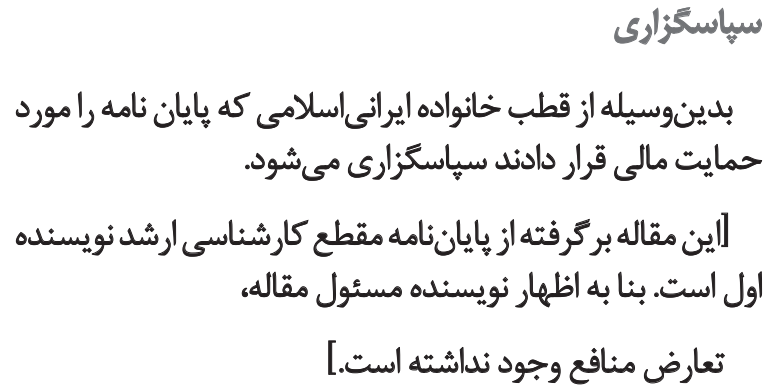
جدينوسيله از قطب خانواده ايرانياسلامى كه بايان نامه را مورد حمايت مالى قرار دادند سياسكزارى مي شئودان

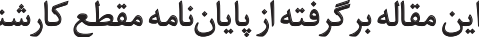
تعارض منافع وجود نداشته است.]


[15] Garrison CZ, Addy CL, Jackson KL, McKeown RE, \& Waller JL. A longitudinal study of suicidal ideation in young adolescents. Journal of the American Academy of Child \& Adolescent Psychiatry. 1991; 30(4):597-603. doi: 10.1097/00004583-199107000-00011

[16] Adams DM, Overholser JC, Lehnert K. Perceived family functioning and adolescent suicidal behavior. Journal of the American Academy of Child \& Adolescent Psychiatry. 1994; 33(4):498-507. doi: 10.1097/00004583-199405000-00008

[17] Maraš JS, Dukić O, Marković J, Biro M. Family and individual factors of suicidal ideation in Adolescents. Psihologija. 2011; 44(3):245-260. doi: 10.2298/psi1103245s

[18] Ryan CE, Epstein NB, Keitner I. Evaluating and treating families: The McMaster approach. Philadelphia: Taylor \& Francis; 2005.

[19] Kabacoff RI, Miller IW, Bishop DS, Epstein NB, Keitner GI. A psychometric study of the McMaster Family Assessment Device in psychiatric, medical, and nonclinical samples. Journal of Family Psychology. 1990; 3(4):431. doi: 10.1037/h0080547

[20] Malek Khosravi, Gh. [Family function of AD/HD children (Persian)] [PhD thesis]. Tehran: University of Social Welfare \& Rehabilitation Sciences; 2003.

[21] Beck AT, Weissman A, Lester D, Trexler L. The measurement of pessimism: The hopelessness scale. Journal of Consulting \& Clinical Psychology. 1974; 42(6):861-65. PMID: 4436473

[22] Beck AT, Steer RA. Manual for the Beck hopelessness scale. San Antonio, TX: Psychological Corporation; 1988.

[23] Perczel Forintos D, Sallai J, Rózsa. Adaptation of the Beck Hopelessness Scale in Hungary. Psychological Topics. 2010; 19(2):307-321.

[24] Hanna D, White R, Lyons K, McParland J, Shannon C, Mulholland C. The structure of the Beck Hopelessness Scale: A confirmatory factor analysis in UK students. Personality and Individual Differences. 2011; 51(1):17-22. doi: 10.1016/j.paid.2011.03.001

[25] Steed L. Further validity and reliability evidence for Beck Hopelessness Scale scores in a nonclinical sample. Educational \& Psychological Measurement. 2001; 61(2):303-16. doi: $10.1177 / 00131640121971121$

[26] Mesbah N, Abedian A. [The relationship of stress and hopelessness among dormitory students (Persian)]. Iranian Journal of Psychiatry \& Clinical Psychology. 2006; 2(45):154-159.

[27] Beck AT, Steer RA. Manual for the Beck scale for suicide ideation. San Antonio, TX: Psychological Corporation; 1991.

[28] Anisi J, Fathi Ashtiani A, Salimi SH, Ahmadi Kh. [Validity and reliability of Beck suicide scale ideation among soldiers (Persian)]. Journal of Military Medicine. 2005; 7(1):33-37.

[29] Hovey JD, Magaña CG. Suicide risk factors among Mexican migrant farm worker women in the Midwest United States. Archives of Suicide Research. 2003; 7(2):107-121. doi: 10.1080/13811110301579

[30] McDermut W, Miller IW, Solomon D, Ryan CE, Keitner GI. Family functioning and suicidality in depressed adults. Comprehensive Psychiatry. 2001; 42(2):96-104. PMID: 11244144

[31] Lipschitz JM, Yen S, Weinstock LM, Spirito A. Adolescent and caregiver perception of family functioning: Relation to suicide ideation and attempts. Psychiatry Research. 2012; 200(2):400-403. doi: 10.1016/j.psychres.2012.07.051

[32] Ören N. Hopelessness levels of children living with their parents or in an orphanage. Self Identity. 2012; 40(3):501-508. doi: 10.2224/sbp.2012.40.3.501

[33] Heikkinen M, Aro H, Lönnqvist J. Recent life events, social support and suicide. Acta Psychiatrica Scandinavica. 1994; 89(377):6572. doi: 10.1111/j.1600-0447.1994.tb05805.x

[34] Heisel MJ, Flett G, Hewitt PL. Social hopelessness and college student suicide ideation. Archives of Suicide Research. 2003; 7(3):221-235. doi: 10.1080/13811110301557

[35] Chioqueta AP, StilesTC. Personality traits and the development of depression, hopelessness, and suicide ideation. Personality \& Individual Differences. 2005; 38(6):1283-1291. doi: 10.1016/j. paid.2004.08.010

[36] Tanaka E, Sakamoto S, Ono Y, Fujihara S, Kitamura T. Hopelessness in a community population: Factorial structure and psychosocial correlates. Journal of Social Psychology. 1998; 138(5):581-590. doi: 10.1080/00224549809600413

[37] Girgin G. Evaluation of the factors affecting loneliness and hopelessness among university students in Turkey. Social Behavior \& Identity. 2009; 37(6):811-818. doi: 10.2224/sbp.2009.37.6.811

[38] Ellis TE. Cognition and suicide: Theory, research, and therapy. Washington, D.C.: American Psychological Association; 2006.

[39] Baron, RM, Kenny DA. The moderator-mediator variable distinction in social psychological research: Conceptual, strategic, and statistical considerations. Journal of Personality \& Social Psychology. 1986; 51(6):1173. doi: 10.1037//0022-3514.51.6.1173 